\title{
Circ-ITCH correlates with less advanced tumor features as well as prolonged survival, and it inhibits cells proliferation but promotes apoptosis in non-small cell lung cancer
}

\author{
Zhenhua Li", Xiaoqun Guo", Shan Gao \\ Department of Pneumology, The Central Hospital of Wuhan, Tongji Medical College, Huazhong University of Science and Technology, Wuhan \\ 430014, China \\ Contributions: (I) Conception and design: S Gao; (II) Administrative support: None; (III) Provision of study materials or patients: All authors; (IV) \\ Collection and assembly of data: Z Li, X Guo; (V) Data analysis and interpretation: Z Li, X Guo; (VI) Manuscript writing: All authors; (VII) Final \\ approval of manuscript: All authors. \\ \#These authors contributed equally to this work. \\ Correspondence to: Shan Gao. Department of Pneumology, The Center Hospital of Wuhan, Tongji Medical College, Huazhong University of Science \\ and Technology, 26 Shengli Street, Wuhan 430014, China. Email: gao_shany@126.com.
}

Background: This study was to investigate the correlation of circ-ITCH expression with tumor features, disease free survival (DFS) and overall survival (OS) in non-small cell lung cancer (NSCLC) patients, and the effect of its dysregulation on cells proliferation and apoptosis of NSCLC cells.

Methods: Tumor tissue and paired adjacent normal tissue samples from 190 surgical NSCLC patients were collected and used for detecting circ-ITCH expression by qPCR. Circ-ITCH expression was also measured in normal pulmonary epithelial cell line and 4 NSCLC cell lines by qPCR. Patients' baseline characteristics were recorded and DFS as well as OS were calculated. Blank, circ-ITCH overexpression, blank shRNA, circITCH shRNA plasmids were transferred into NSCLC cell line (A549 cells) as NC(+), Circ-ITCH(+), NC(-) and $\mathrm{Circ-ITCH}(-)$ groups. Cells proliferation was measured using CCK-8 and cells apoptosis rate was measured using annexin $\mathrm{V}(\mathrm{AV})$ /propidium iodide $(\mathrm{PI})$.

Results: Circ-ITCH expression was decreased in tumor tissue compared with paired adjacent tissue, and was negatively correlated with tumor size, lymph node metastasis and TNM stage. And circ-ITCH high expression was associated with favorable DFS and OS. In vitro experiments revealed that compared with normal pulmonary epithelial cell line BEAS-2B, circ-ITCH expression was reduced in NSCLC cell lines HCC827, A549, NCI-H1299 and NCI-H460. And cells proliferation was decreased in Circ-ITCH(+) group and elevated in Circ-ITCH(-) group at $72 \mathrm{~h}$. Cells apoptosis rate was increased in Circ-ITCH(+) group and decreased in Circ-ITCH(-) group at $72 \mathrm{~h}$.

Conclusions: Circ-ITCH associates with less advanced tumor features and prolonged survival, and it inhibits cells proliferation while promotes cells apoptosis in NSCLC.

Keywords: Circ-ITCH; non-small cell lung cancer (NSCLC); survival; cell proliferation; cell apoptosis

Submitted Sep 29, 2018. Accepted for publication Dec 21, 2018.

doi: $10.21037 /$ tcr.2019.08.01

View this article at: http://dx.doi.org/10.21037/tcr.2019.08.01

\section{Introduction}

Lung cancer is the most frequently diagnosed cancer and the leading cause of cancer-related death not only in China but also around the world $(1,2)$. As a developing country,
China undergoes rapid elevation of incidence and mortality of lung cancer during the past three decades, which is on the contrary to most developed countries $(2,3)$. Nonsmall cell lung cancer (NSCLC) is the most common type 
of lung cancer and approximately accounts for $85 \%$ of all lung cancer cases, also it is widely accepted that NSCLC is the most prevalent type of lung cancer in China (1,4-6). Although various approaches have been introduced for the treatment of NSCLC, such as anti-angiogenesis agents and tyrosine kinase (TK) inhibitors of the epidermal growth factor receptor (EGFR), the prognosis of NSCLC is still unsatisfying. Therefore, it is necessary to explore more biomarkers to monitor the development of NSCLC and guide clinical management.

Circular RNA (circRNA) is a novel class of endogenous RNAs with covalently closed continuous loop and is revealed to be closely related to tumor development and progression (7-9). Circ-ITCH is a reported circRNA generated from several exons of itchy E3 ubiquitin protein ligase (ITCH) and plays as a tumor suppressor gene in several types of carcinomas including bladder cancer, colorectal cancer, esophageal squamous cell carcinoma (ESCC) and hepatocellular carcinoma (10-13). As to lung cancer, only one study showed that circ-ITCH is downregulated in lung cancer cell lines which inhibits cells proliferation via regulating $\mathrm{Wnt} / \beta$-Catenin pathway, however, to our best knowledge, there are still no other reported studies on the functional roles of circ-ITCH in NSCLC until now (14). Thus, the objective of this study was to investigate the correlation of circ-ITCH expression with tumor features, disease free survival (DFS) and overall survival (OS) in NSCLC patients, and the effect of its dysregulation on cells proliferation and apoptosis of NSCLC cells.

\section{Methods}

\section{Patients, samples and measurement of circ-ITCH expression}

A total of 190 primary NSCLC patients underwent surgery at The Central Hospital of Wuhan, Tongji Medical College, Huazhong University of Science and Technology between January 2014 and December 2014 were prospectively enrolled in this study. Tumor tissue and paired adjacent normal tissue samples were collected during the surgery and immediately stored in liquid nitrogen. Circ-ITCH expression in tumor and paired adjacent samples were measured using quantitative polymerase chain reaction (qPCR). This study was approved by the Ethics Review Board of The Central Hospital of Wuhan, Tongji Medical College, Huazhong University of Science and Technology, and all patients signed the informed consents. The Number of the Ethic Approval was (2013)-Ethical Approved-(03).

\section{Patients data collection and follow-ups}

Key features of NSCLS patients at baseline were documented including age, gender, pathological grade, tumor size, status of lymph node metastasis, status of distant metastasis and TNM stage. Patients were regularly followups by visits or phone 1 time every month in the first half year, and then 1 time every 3-6 months in the remaining follow-up duration, and all patients were followed up for 36 months with the last follow up date $2017 / 12 / 31$. DFS and OS were calculated.

\section{Measurement of circ-ITCH expression in NSCLC cell lines}

Normal pulmonary epithelial cell line BEAS-2B and NSCLC cell lines HCC827, A549, NCI-H1299, NCI-H460 were purchased from Cell Resource Center of Shanghai Institute of Life Sciences, Chinese Academy of Sciences (Shanghai, China). BEAS-2B cells were cultured in $90 \%$ MEM medium (Gibco, USA) supplemented with $10 \%$ fetal bovine serum (FBS) (Gibco, USA). HCC827, NCI-H1299 and NCI-H460 cells were cultured in 90\% RPMI medium (Gibco, USA) supplemented with 10\% FBS (Gibco, USA), A549 cells were cultured in 90\% F12K medium (Gibco, USA) supplemented with $10 \%$ FBS (Gibco, USA). All cells were incubated under $95 \%$ air and $5 \% \mathrm{CO}_{2}$ at $37{ }^{\circ} \mathrm{C}$. CircITCH expression in normal pulmonary epithelial cell line and NSCLC cell lines were then measured using qPCR.

\section{Measurement of the effect of circ-ITCH overexpression and shRNA on A549 cells functions}

Blank plasmids, circ-ITCH overexpression plasmids, blank shRNA plasmids, circ-ITCH shRNA plasmids were constructed by Shanghai GenePharma Company (Shanghai, China) and transferred into A549 cells as $\mathrm{NC}(+)$, CircITCH(+), NC(-) and circ-ITCH(-) groups. Subsequently, circ-ITCH expression was measured using qPCR at $24 \mathrm{~h}$, and cells proliferation was measured using CCK-8 at 0, 24, 48 and $72 \mathrm{~h}$, while cells apoptosis rate was measured using annexin $\mathrm{V}(\mathrm{AV})$ /propidium iodide (PI) at $72 \mathrm{~h}$.

\section{qPCR}

Total RNA was extracted from tissue samples or cells using 
TRIzol Reagent (Invitrogen, USA). Then 1 microgram ( $\mu \mathrm{g})$ RNA was reversely transcribed to cDNA using ReverTra Ace ${ }^{\circledR}$ qPCR RT Master Mix (Toyobo, Japan), and qPCR was carried out using QuantiNova SYBR Green PCR Kit (Qiagen, German) to determine the expression of glyceraldehyde3-phosphate dehydrogenase (GAPDH). Subsequently, 1 ug RNA was digested using RNase R (Epicentre, USA) to eliminate linear RNA, and then reversely transcribed to cDNA using PrimeScript ${ }^{\mathrm{TM}} \mathrm{RT}$ reagent Kit (Takara, Japan), and qPCR was carried out using the QuantiNova SYBR Green PCR Kit (Qiagen, German) to determine the expression of circ-ITCH. The primer of GAPDH was: forward 5' GAGTCCACTGGCGTCTTCAC 3', reverse 5' ATCTTGAGGCTGTTGTCATACTTCT 3'; the primer of circ-ITCH was: forward 5' GCAGAGGCCAACACTGGAA 3', reverse 5' TCCTTGAAGCTGACTACGCTGAG 3'. The expression of circ-ITCH was then calculated using $2^{-\Delta \Delta C t}$ formula with GAPDH as internal reference.

\section{CCK8 Cell proliferation assay}

To perform cell proliferation assay, the A549 cells were seeded into 96-well plates at a density of 2,000 cells per well. After transfection of the corresponding plasmid, cells were incubated for 24,48 , and $72 \mathrm{~h}$. Cell viability was measured by the cell counting kit-8 (CCK-8) (Dojindo, Japan) at $0,24,48$ and $72 \mathrm{~h}$ according to the manufacturer's instructions. Absorbance at $450 \mathrm{~nm}$ of each well was measured by using microplate reader (BioTek, USA). Each group had 3 replicates and the measurement of absorbance was repeated at least 3 times.

\section{Apoptosis measurement}

To detect cell apoptosis, AV/PI apoptosis detection kit (Beyotime, China) was used according to the manufacturer's recommendations. After $48 \mathrm{~h}$ transfection of the corresponding plasmid, the cells were harvested and then stained with AVFITC and PI in the dark $25^{\circ} \mathrm{C}$ for $15 \mathrm{~min}$. AV and PI labeling were measured by a flow cytometer (BD, USA).

\section{Statistics}

Statistical analysis was performed using SPSS 22.0 Software (IBM, USA) and graphs were made using GraphPad Software 6.01 (GraphPad Software, USA). Data were presented as mean \pm standard deviation or standard error (SEM), or median $\left(25^{\text {th }}-75^{\text {th }}\right.$ quantiles $)$ or count
(\%). Comparison between paired groups was detected by Wilcoxon signed rank sum test, comparison between individual groups was detected by $t$ test, Wilcoxon rank sum test or Chi-square test. Kaplan-Meier (K-M) curve and logrank test were used to compare DFS and OS between two groups. $\mathrm{P}<0.05$ was considered as significant.

\section{Results}

\section{Characteristics of NSCLC patients}

The mean age of NSCLC patients was $61.9 \pm 9.9$ years and there were 103 males as well as 87 females enrolled in this study. As for the pathological grade, there were 26 $(13.7 \%)$ patients with well differentiation, $122(64.2 \%)$ patients with intermediate differentiation and $42(22.1 \%)$ patients with poor differentiation. The numbers of patients with tumor size $\leq 5$ or $>5 \mathrm{~cm}$ were $105(55.3 \%)$ and 85 (44.7\%), respectively. As to lymph node metastasis, 116 (61.1\%) patients were negative and 74 (38.9\%) patients were positive. All patients were diagnosed without distant metastasis. In addition, there were 61 (32.1\%), 64 (33.7\%) and $65(34.2 \%)$ patients distributed in TNM stage I, II and III, respectively (Table 1).

\section{Comparison of circ-ITCH expression between tumor tissue and paired adjacent tissue}

Wilcoxon signed rank sum test was performed to evaluate the difference of circ-ITCH expression between paired adjacent tissue and tumor tissue, which disclosed that the circ-ITCH expression was down-regulated in tumor tissue compared with paired adjacent tissue $(\mathrm{P}<0.001)$ (Figure 1).

\section{Circ-ITCH expression correlated with tumor features}

In order to better understand the correlation of circ-ITCH with clinicopathological characteristics and prognosis in NSCLC patients, we divided NSCLC patients into circ-ITCH low expression group or circ-ITCH high expression group according to the median value of circITCH expression level in NSCLC patients as cut off value of 0.451. And NSCLC patients with circ-ITCH expression less than 0.451 were assigned in circ-ITCH low expression group, while NSCLC patients with circ-ITCH expression more than 0.451 were assigned in circ-ITCH high expression group. Chi-square test and Wilcoxon rank sum test were used to compare the difference of tumor features 
Table 1 Characteristics of NSCLC patients

\begin{tabular}{|c|c|}
\hline Parameters & NSCLC patients $(\mathrm{N}=190)$ \\
\hline Age (years) & $61.9 \pm 9.9$ \\
\hline \multicolumn{2}{|l|}{ Gender (n/\%) } \\
\hline Male & $103(54.2)$ \\
\hline Female & $87(45.8)$ \\
\hline \multicolumn{2}{|l|}{ Pathological grade (n/\%) } \\
\hline Well differentiation & $26(13.7)$ \\
\hline Intermediate differentiation & $122(64.2)$ \\
\hline Poor differentiation & $42(22.1)$ \\
\hline \multicolumn{2}{|l|}{ Tumor size (n/\%) } \\
\hline$\leq 5 \mathrm{~cm}$ & $105(55.3)$ \\
\hline$>5 \mathrm{~cm}$ & $85(44.7)$ \\
\hline \multicolumn{2}{|l|}{ Lymph node metastasis (n/\%) } \\
\hline Negative & $116(61.1)$ \\
\hline Positive & $74(38.9)$ \\
\hline \multicolumn{2}{|l|}{ Distant metastasis (n/\%) } \\
\hline Negative & $190(100.0)$ \\
\hline Positive & $0(0.0)$ \\
\hline \multicolumn{2}{|l|}{ TNM stage (n/\%) } \\
\hline I & $61(32.1)$ \\
\hline II & $64(33.7)$ \\
\hline III & $65(34.2)$ \\
\hline
\end{tabular}

Data were presented as mean \pm standard deviation or count (\%). NSCLC, non-small-cell lung carcinoma.

between circ-ITCH high expression group and circ-ITCH low expression group, which revealed that patients in circITCH high expression group presented with smaller tumor size $(\mathrm{P}<0.001)$, less lymph node metastasis $(\mathrm{P}<0.001)$ and lower grade of TNM stage $(\mathrm{P}=0.003)$ compared with those in circ-ITCH low expression group (Table 2).

\section{Comparison of DFS and OS between circ-ITCH low expression group and circ-ITCH high expression group}

$\mathrm{K}-\mathrm{M}$ curve and log-rank test were carried out to assess the DFS and OS between circ-ITCH high expression group and circ-ITCH low expression group. Patients in circ-ITCH high expression group had better DFS compared with circ-

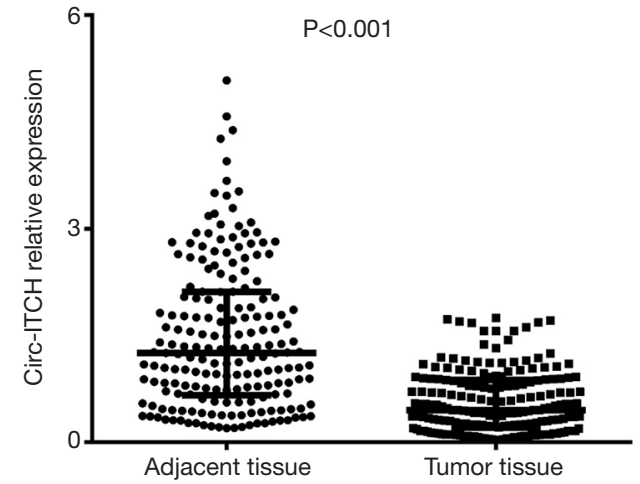

Figure 1 Circ-ITCH expression in paired adjacent tissue and tumor tissue from NSCLC patients. Circ-ITCH expression was decreased in tumor tissue compared with paired adjacent tissue. Wilcoxon signed rank sum test was used to assess the difference of circ-ITCH expression between paired adjacent tissue and tumor tissue. $\mathrm{P}<0.05$ was considered significant. Circ-ITCH, circular RNA-itchy E3 ubiquitin protein ligase; NSCLC, non-small cell lung carcinoma.

ITCH low expression group $(\mathrm{P}=0.001)$. Similarly, favorable OS was displayed in circ-ITCH high expression group than that in the low expression group $(\mathrm{P}=0.006)$ (Figure 2).

\section{Comparison of circ-ITCH expression between normal pulmonary epithelial cell line BEAS-2B and NSCLC cell lines HCC827, A549, NCI-H1299 and NCI-H460}

Compared with normal pulmonary epithelial cell line BEAS-2B, circ-ITCH expression was reduced in NSCLC cell lines including HCC827 $(\mathrm{P}<0.05)$, A549 $(\mathrm{P}<0.01)$, NCI-H1299 $(\mathrm{P}<0.05)$ and NCI-H460 $(\mathrm{P}<0.05)$ (Figure 3$)$.

\section{Circ-ITCH suppressed cells proliferation of A549 cells}

Circ-ITCH expression was higher in circ-ITCH(+) group compared with $\mathrm{NC}(+)$ group $(\mathrm{P}<0.001)$, while decreased circ-ITCH expression was displayed in circ-ITCH(-) group compared with $\mathrm{NC}(-)$ group $(\mathrm{P}<0.001)$, which suggested that circ-ITCH overexpression and shRNA in A549 cells were successfully constructed and transfected (Figure 4A). Cells proliferation was reduced in circ-ITCH $(+)$ group at $48 \mathrm{~h}(\mathrm{P}<0.05)$ and $72 \mathrm{~h}(\mathrm{P}<0.01)$ compared with $\mathrm{NC}(+)$ group, while it was elevated in circ-ITCH(-) group at $48 \mathrm{~h}(\mathrm{P}<0.05)$ and $72 \mathrm{~h}(\mathrm{P}<0.01)$ compared with $\mathrm{NC}(-)$ group (Figure $4 B)$. 
Table 2 Correlation of circ-ITCH expression with tumor features

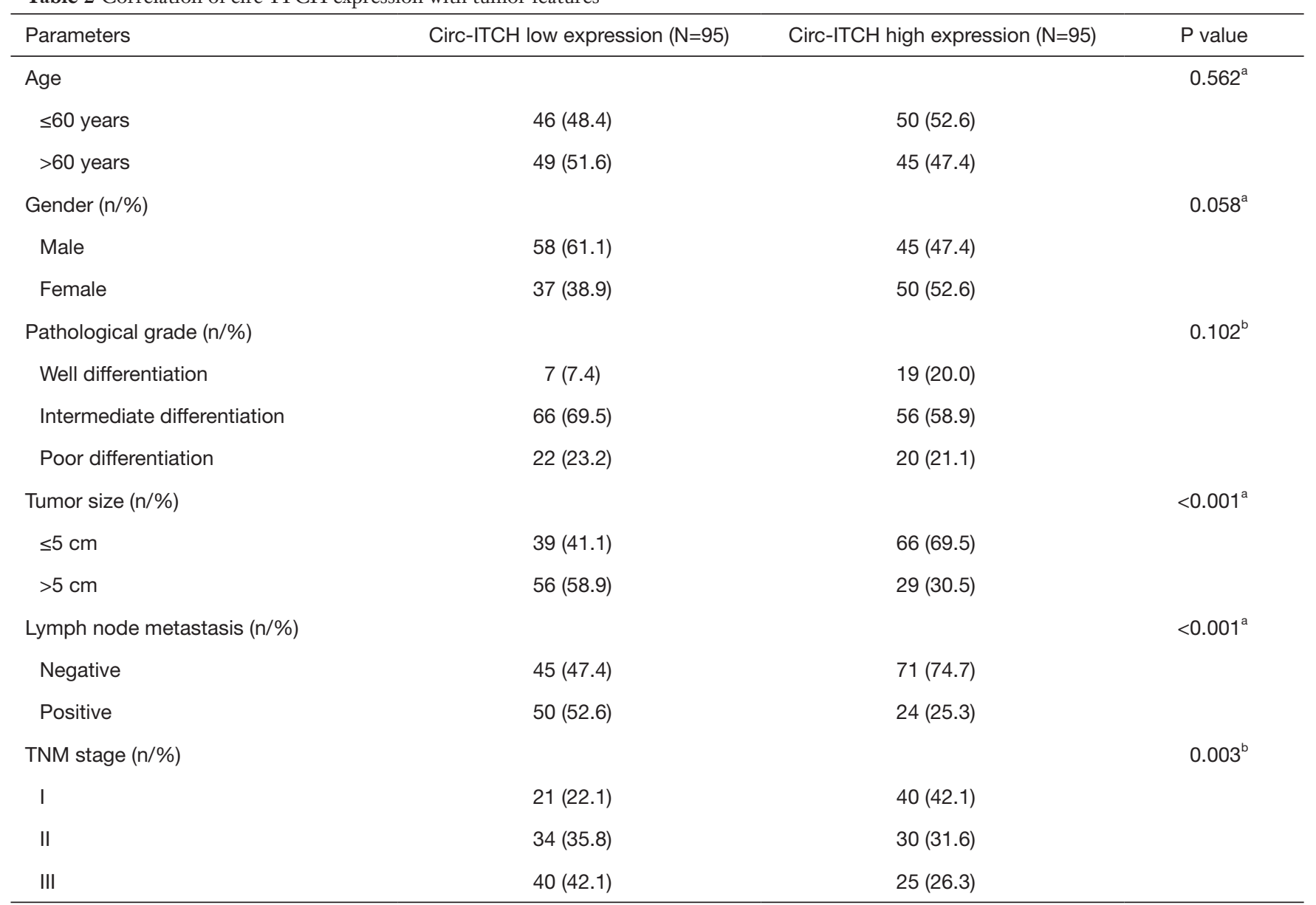

Data were presented as count (\%). ${ }^{\text {, }}$, comparison was detected by Chi-square test; ${ }^{\mathrm{b}}$, comparison was detected by Wilcoxon rank sum test. $\mathrm{P}<0.05$ was considered as significant. Circ-ITCH, circular RNA itchy E3 ubiquitin protein ligase.
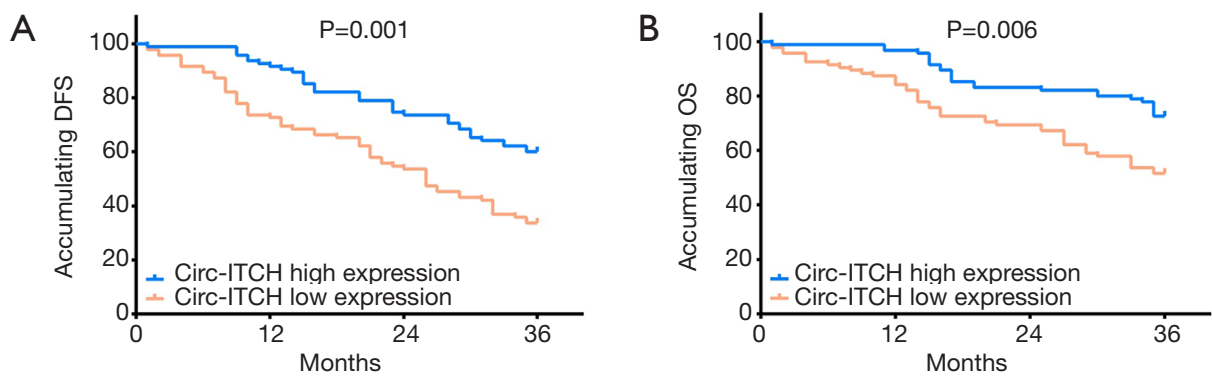

Figure 2 Correlation of circ-ITCH expression with DFS and OS in NSCLC patients. High expression of circ-ITCH was associated with prolonged DFS (A) and OS (B). Kaplan-Meier curves were used to evaluate the correlation of circ-ITCH expression with DFS and OS. Comparison between two groups was analyzed by log-rank test. $\mathrm{P}<0.05$ was considered significant. Circ-ITCH, circular RNA-itchy E3 ubiquitin protein ligase; DFS, disease free survival; OS, overall survival; NSCLC, non-small cell lung carcinoma. 


\section{Circ-ITCH promoted cells apoptosis of A549 cells}

Cells apoptosis rate was increased in circ-ITCH(+) group compared with $\mathrm{NC}(+)$ group $(\mathrm{P}<0.001)$, while it was decreased in circ-ITCH(-) group compared with $\mathrm{NC}(-)$ group $(\mathrm{P}<0.01)$ (Figure $5 A, B)$.

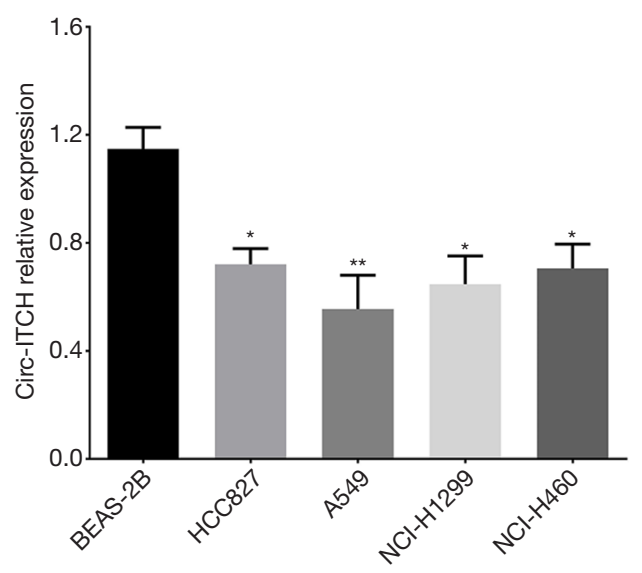

Figure 3 Difference of circ-ITCH expression level between normal pulmonary epithelial cell line and 4 NSCLC cell lines. The level of circ-ITCH expression was lower in NSCLC cell lines HCC827, A549, NCI-H1299 and NCI-H460 compared with normal pulmonary epithelial cell line BEAS-2B. T-test was used to compare the level of circ-ITCH expression between normal pulmonary epithelial cell line BEAS-2B and NSCLC cell lines HCC827, A549, NCI-H1299, NCI-H460. *, $\mathrm{P}<0.05$; ** $\mathrm{P}<0.01$. Circular RNA-itchy E3 ubiquitin protein ligase; NSCLC, nonsmall cell lung carcinoma.

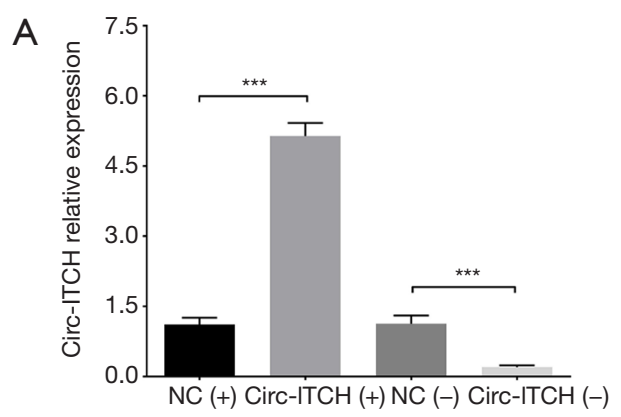

\section{Discussion}

In this study, we observed that: (I) circ-ITCH expression was decreased in tumor tissue compared with paired adjacent tissue, and its high expression correlated with less advanced tumor features, prolonged DFS and OS; (II) circ-ITCH expression was reduced in NSCLC cell lines compared to normal pulmonary epithelial cell line, and it inhibited cells proliferation as well as facilitated cells apoptosis of A549 cells.

Several studies reveal that circ-ITCH expression is reduced not only in tumor tissues but also in cancer-related cell lines and its high expression is correlated with less advanced clinicopathological features of various cancers (10-13). An interesting study illustrates that circ-ITCH expression is decreased in bladder cancer tissues compared with paired adjacent normal tissues and in eight bladder cancer cell lines (EJ, T24, 253 J, RT4, TCC-SUP, UMUC, J82, 5637) compared with normal urothelial cell line SV-HUC (10). Additionally, in their study, the level of circ-ITCH expression in tumor tissue is negatively associated with histological grade in bladder cancer patients (10). Besides, the downregulation of circ-ITCH has also been shown in other carcinomas, such as, colorectal cancer, ESCC and hepatocellular carcinoma (11-13). As to the prognostic value of circ-ITCH expression in cancers, a study observes that bladder cancer patients with circ-ITCH high expression exhibited longer OS with maximal 60 months follow-up duration (10), another study illuminates that hepatocellular carcinoma patients with circ-ITCH high expression also present with favorable OS with maximal follow-up reaching to 7 years (13). In line with these previous studies, we discovered that circ-ITCH expression was

B

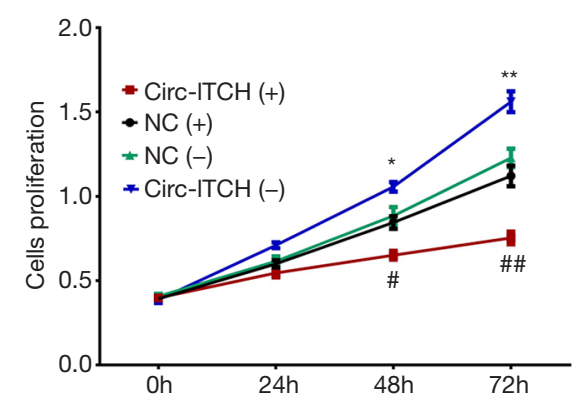

Figure 4 Cells proliferation in A549 cells after transfections. After transfections, elevated circ-ITCH expression level in circ-ITCH(+) group and reduced circ-ITCH expression level in circ-ITCH(-) group were discovered at $24 \mathrm{~h}$ (A). Cells proliferation was reduced in circITCH(+) group and increased in circ-ITCH(-) group at $72 \mathrm{~h}(\mathrm{~B}) . T$-test was used to compare the level of circ-ITCH expression between two groups. NC(+): A549 transfected with blank plasmids; circ-ITCH(+): A549 transfected with circ-ITCH overexpression plasmids; NC(-): A549 transfected with blank shRNA plasmids; circ-ITCH(-): A549 transfected with circ-ITCH shRNA plasmids. *, $\mathrm{P}<0.05$; **, $\mathrm{P}<0.01$; ***, $\mathrm{P}<0.001 ;{ }^{\#}, \mathrm{P}<0.05 ;{ }^{\# \#}, \mathrm{P}<0.01$. Circ-ITCH, circular RNA-itchy E3 ubiquitin protein ligase. 
A

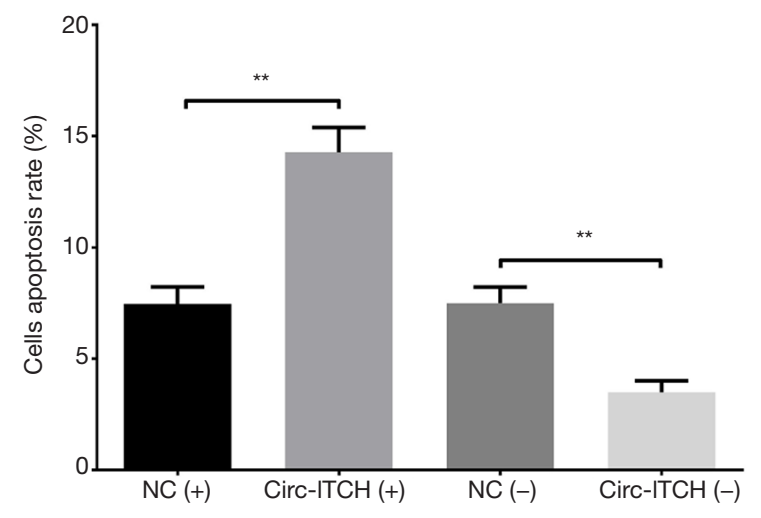

B

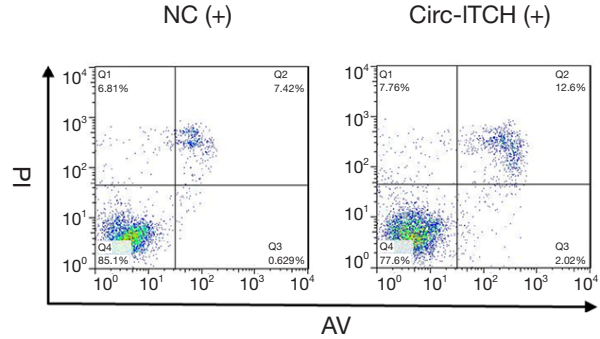

$\mathrm{NC}(-)$

Circ-ITCH (-)

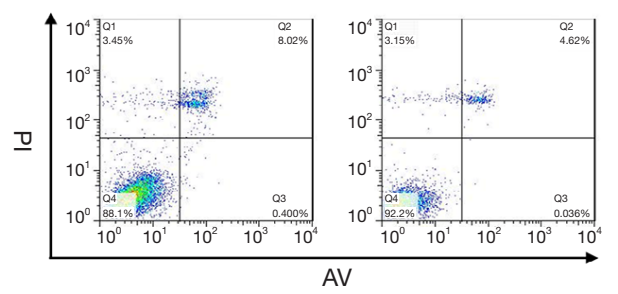

Figure 5 Cells apoptosis in A549 cells after transfections. After transfections, cells apoptosis rate at $72 \mathrm{~h}$ was increased in circ-ITCH(+) group compared with $\mathrm{NC}(+)$ group (A), and decreased in circ-ITCH(-) compared with $\mathrm{NC}(-)$ group (B). $T$-test was used to compare the rates of cells apoptosis in $\mathrm{NC}(+)$, circ-ITCH(+), $\mathrm{NC}(-)$ and circ-ITCH(-) between two groups. **, $\mathrm{P}<0.01$. Circ-ITCH, circular RNA-itchy E3 ubiquitin protein ligase.

downregulated in tumor tissues compared with paired adjacent tissue, and its high expression was associated with smaller tumor size, less lymph node metastasis and lower TNM stage, and better DFS as well as OS. The possible explanation for our results could be that: circ-ITCH was enriched with miRNA binding sites that bound to several tumor promoter miRNAs, such as miR-196b, miR-150 and miR-182, leading to overexpressed ITCH and accelerated degradation of disheveled (Dvl) via regulating multiple pathways, for example inhibiting the $\mathrm{Wnt} / \beta$-catenin pathway, thereby suppressing cancer cells proliferation, migration, invasion and metastasis and resulting in less progressive clinicopathological features as well as prolonged DFS/OS (10-13,15-18). Moreover, our further cellular experiments revealed the role of circ-ITCH in mediating cells functions of NSCLC cells, which could also provide some evidence to these clinical findings. In addition, there was a limitation in this study: the circ-ITCH expression level was detected in tissue samples instead of blood samples in our study, if considering circ-ITCH as a biomarker to monitor cancer development and progression, blood sample would be more accessible which needed to be further explored.

Recent studies elucidate that circ-ITCH is involved in the regulation of cancer cells functions, including proliferation, migration, invasion, metastasis and apoptosis (10-13). A study reveals that circ-ITCH decreased cells viability, but enhanced G1/S cell cycle arrest and cells apoptosis in bladder cancer cell lines EJ and T24 (10). Another study discloses that circ-ITCH inhibits cancer cells proliferation by repressing $\mathrm{Wnt} / \beta$-catenin pathway in colorectal cancer (11). Moreover, circ-ITCH represses cancer cells proliferation probably via regulating ubiquitination and degradation of phosphorylated Dvl2 to suppress the Wnt/ $\beta$-catenin pathway in ESCC (12). These previous studies suggest the inhibitory role of circ-ITCH in carcinogenesis and cancer progression through suppressing cancer cells proliferation and enhancing cells apoptosis, as for our study, the results revealed that circ-ITCH expression was decreased in four NSCLC cell lines (HCC827, A549, NCI-H1299 and NCI-H460) compared with normal pulmonary epithelial cell line (BEAS-2B), and it participated in inhibition of cells proliferation and promotion of cells apoptosis of NSCLC cells, which indicated that circ-ITCH played an inhibitory role in development and progression of NSCLC. These results from cells experiments in our study could be explained by that: circITCH might mediate cells functions through binding to many pro-tumorigenesis miRNAs including miR-7, miR-17 and miR-214 and subsequently modulating various pathways, such as suppressing the $\mathrm{Wnt} / \beta$-catenin pathway (10-13).

\section{Conclusions}

In brief, circ-ITCH associates with less advanced tumor 
features and prolonged survival as well as inhibits cells proliferation while promotes cells apoptosis in NSCLC.

\section{Acknowledgments}

Funding: None.

\section{Footnote}

Conflicts of Interest: All authors have completed the ICMJE uniform disclosure form (available at http://dx.doi. org/10.21037/tcr.2019.08.01). The authors have no conflicts of interest to declare.

Ethical Statement: The authors are accountable for all aspects of the work in ensuring that questions related to the accuracy or integrity of any part of the work are appropriately investigated and resolved. The study was conducted in accordance with the Declaration of Helsinki (as revised in 2013). This study was approved by the Ethics Review Board of The Central Hospital of Wuhan, Tongji Medical College, Huazhong University of Science and Technology, and all patients signed the informed consents. The Number of the Ethic Approval was (2013)-Ethical Approved-(03).

Open Access Statement: This is an Open Access article distributed in accordance with the Creative Commons Attribution-NonCommercial-NoDerivs 4.0 International License (CC BY-NC-ND 4.0), which permits the noncommercial replication and distribution of the article with the strict proviso that no changes or edits are made and the original work is properly cited (including links to both the formal publication through the relevant DOI and the license). See: https://creativecommons.org/licenses/by-nc-nd/4.0/.

\section{References}

1. Zhou C. Lung cancer molecular epidemiology in China: recent trends. Transl Lung Cancer Res 2014;3:270-9.

2. Torre LA, Bray F, Siegel RL, et al. Global cancer statistics, 2012. CA Cancer J Clin 2015;65:87-108.

3. Siegel RL, Miller KD, Jemal A. Cancer Statistics, 2017. CA Cancer J Clin 2017;67:7-30.

4. Molina JR, Yang P, Cassivi SD, et al. Non-small cell lung cancer: epidemiology, risk factors, treatment, and survivorship. Mayo Clin Proc 2008;83:584-94.

5. Navada S, Lai P, Schwartz AG, et al. Temporal trends in small cell lung cancer: Analysis of the national Surveillance,
Epidemiology, and End-Results (SEER) database. J Clin Oncol 2006;24:abstr 7082.

6. Sher T, Dy GK, Adjei AA. Small cell lung cancer. Mayo Clin Proc 2008;83:355-67.

7. Memczak S, Jens M, Elefsinioti A, et al. Circular RNAs are a large class of animal RNAs with regulatory potency. Nature 2013;495:333-8.

8. Qu S, Yang X, Li X, et al. Circular RNA: A new star of noncoding RNAs. Cancer Lett 2015;365:141-8.

9. Li W, Zheng J, Deng J, et al. Increased levels of the long intergenic non-protein coding RNA POU3F3 promote DNA methylation in esophageal squamous cell carcinoma cells. Gastroenterology 2014;146:1714-26.e5.

10. Yang C, Yuan W, Yang X, et al. Circular RNA circ-ITCH inhibits bladder cancer progression by sponging miR17/miR-224 and regulating p21, PTEN expression. Mol Cancer 2018;17:19.

11. Huang G, Zhu H, Shi Y, et al. cir-ITCH plays an inhibitory role in colorectal cancer by regulating the Wnt/ beta-catenin pathway. PLoS One 2015;10:e0131225.

12. Li F, Zhang L, Li W, et al. Circular RNA ITCH has inhibitory effect on ESCC by suppressing the Wnt/betacatenin pathway. Oncotarget 2015;6:6001-13.

13. Guo W, Zhang J, Zhang D, et al. Polymorphisms and expression pattern of circular RNA circ-ITCH contributes to the carcinogenesis of hepatocellular carcinoma. Oncotarget 2017;8:48169-77.

14. Wan L, Zhang L, Fan K, et al. Circular RNA-ITCH Suppresses Lung Cancer Proliferation via Inhibiting the Wnt/ beta-Catenin Pathway. Biomed Res Int 2016;2016:1579490.

15. Li H, Feng C, Shi S. miR-196b promotes lung cancer cell migration and invasion through the targeting of GATA6. Oncol Lett 2018;16:247-52.

16. Jiang K, Shen M, Chen Y, et al. miR150 promotes the proliferation and migration of nonsmall cell lung cancer cells by regulating the SIRT2/JMJD2A signaling pathway. Oncol Rep 2018;40:943-51.

17. Chang H, Liu YH, Wang LL, et al. MiR-182 promotes cell proliferation by suppressing FBXW7 and FBXW11 in nonsmall cell lung cancer. Am J Transl Res 2018;10:1131-42.

18. Gao C, Chen YG. Dishevelled: The hub of Wnt signaling. Cell Signal 2010;22:717-27.

Cite this article as: $\mathrm{Li}$ Z, Guo X, Gao S. Circ-ITCH correlates with less advanced tumor features as well as prolonged survival, and it inhibits cells proliferation but promotes apoptosis in nonsmall cell lung cancer. Transl Cancer Res 2019;8(5):1672-1679. doi: 10.21037/tcr.2019.08.01 